\title{
Retrieval processes in distance cognition
}

\author{
EDWARD K. SADALLA, LORIN J. STAPLIN, and W. JEFFREY BURROUGHS \\ Arizona State University, Tempe, Arizona 85281
}

\begin{abstract}
Two studies were performed to evaluate the role of information retrieval in the cognition of traversed distance. In Study 1, subjects walked a pathway containing intersections that were labeled with either high-frequency or low-frequency proper names. The pathway with high-frequency names was estimated as longer than the pathway with low-frequency names. Subsequent tests of memory for names indicated that high- and low-frequency names were recognized equally well, but that the former were recalled more easily. Prompting subjects with intersection names eliminated the difference in distance estimation between highand low-frequency name conditions. A second study demonstrated that category prompts that increased information recall also increased estimated distance. Results were interpreted as suggesting a significant role of information retrieval in distance cognition.
\end{abstract}

One of the most ancient and most functional of human abilities consists of the capacity to estimate how far one has traveled during a journey. Until the recent interest in the topic of cognitive mapping (Downs \& Stea, 1973, 1977; Kosslyn, Pick, \& Fariello, 1974; Siegel \& White, 1975), this ability has largely escaped the scrutiny of experimental psychologists. Cognitive mapping generally refers to the process by which individuals collect, organize, store, retrieve, and manipulate information concerning location in space. The research reported below explores the role of two of these operations, storage and retrieval, on the estimation of traversed distance.

The study of the cognition of traversed distance examines distance estimates that are made in the absence of direct sensory referents. Distances are estimated either at a point removed in time or on a scale too large to lie within the immediate sensory field of an observer. In such contexts individuals are required to integrate a series of perceptual events into an estimate of traversed distance. The paradigm is designed to be representative of the task facing an individual who is trying to cognize the spatial relationships between locations in a novel architectural or urban environment.

Milgram (1973) has suggested that the cognitive representation of large-scale space is influenced by the amount of information that an individual has stored about a given region. He suggests that "size distortions in mental maps may in some way represent an amount proportional to the molecular storage units used for different realms of the environment" (Milgram, 1973, p. 23). This concept of the amount of information in storage may account for the well documented tendency for individuals to represent known regions as larger than unknown regions in map drawing tasks (Downs \& Stea, 1973; Gould \& White, 1974; Stea, 1969). A similar "storage size hypothesis" has been suggested to account for data in studies of temporal duration. Temporal intervals that contain more information are reliably estimated as longer than equal intervals that contain less information (Ornstein, 1969).

The information storage metaphor also appears to account for data that demonstrate a relationship between the structure of a traversed route and the estimated length of that route. A number of studies have indicated that the number of turns that are distributed along a route affects the perceived length of the route. Routes with more turns are generally estimated as longer than routes of equivalent length that contain fewer turns (Downs \& Stea, 1973; Lee, 1970; Sadalla \& Magel, in press). Estimated route length is also influenced by the number of intersections that are distributed along a route; routes with more intersections are consistently estimated as longer than routes of equivalent objective distance with fewer intersections (Sadalla \& Staplin, in press).

If structural changes in a route tend to be remembered at tributes of a route, then previous studies that demonstrate an impact of turns and intersections on route distance estimation may be interpreted as supporting an information storage model. Since such a model predicts increases in cognized distance with increases in stored route attributes, it may account for effects observed in the previous studies.

The two experiments described below were designed to evaluate the effect of memory for route attributes on distance estimation. The common distinction drawn between information storage and information retrieval (Tulving \& Pearlstone, 1966) has been operationalized by assuming that recognition memory tasks primarily assess what is in storage, while recall tasks reflect the additional effect of retrieval operations (Kintsch, 1970; Slamecka, 1968; Tulving \& Osler, 1968). The specific question addressed in the following studies is as follows: Is the estimation of traversed distance influenced by the amount of stored route information or by the amount of such information that can be retrieved? This question is examined by arranging conditions in which the amount 
of stored route information is equivalent for all subjects, but in which the retrieval of route information is easier for some subjects than for others. If distance cognition is influenced primarily by information storage, then manipulations affecting retrieval should not affect distance estimates. Conversely, if the experimental manipulations do affect distance cognition, then the retrieval of route information is implicated as a crucial process in distance cognition.

In Experiment 1, subjects traversed an otherwise featureless path containing 15 intersections that were labeled with eight high-frequency or low-frequency proper names. Memory for route attributes was assessed by both recall and recognition tests, and distance estimates were collected. It was assumed that high-frequency names would be easier to recall (Deese, 1960; Hall, 1954) and that increased recall would be associated with increased distance estimates. Recognition tests were included to evaluate whether information storage per se plays a role in distance cognition.

\section{EXPERIMENT 1}

\section{Method}

Subjects. Subjects were 96 students from introductory and environmental psychology classes at Arizona State University, who partially fulfilled course requirements by participating in this experiment. An approximately equal number of males and females served as subjects in a between-subjects design.

Apparatus. The apparatus used in this study consisted of a pathway marked with 4-cm-wide masking tape on the floor of a large $(12 \mathrm{~m} \mathrm{x} 17 \mathrm{~m})$ empty room. The pathway consisted of two 9-m linear segments that were joined at a 90-deg angle. The two segments contained eight and seven intersections, respectively, formed by additional strips of tape that all intersected the pathway at $90-\mathrm{deg}$ angles. Two sets of name cards, which were used to label the 15 intersections, were constructed by lettering 15 high-frequency and 15 low-frequency proper names in black ink on $30 \times 6 \mathrm{~cm}$ pieces of white posterboard. As a further control, each high-frequency name was matched with a lowfrequency name that began with the same letter and contained the same number of letters and syllables. Table 1 presents the two sets of proper names used in the study, showing the frequency of occurrence of each name per 100,000 words in the English language (Carroll, Davies, \& Richman, 1971). Each name placard was placed on the floor overlying the intersecting strips of tape and immediately to the right of the traversed pathway. The position of all high-frequency names in the 15-name sequence was determined using a random number program, and the same sequence was maintained for matched low-frequency names in the other experimental condition.

The final item of apparatus used in this study was a specially designed headpiece with an adjustable horizontal blinder. This was worn by each subject during the pathway traversal, in an effort to restrict the forward visual field of the subject to approximately $1 \mathrm{~m}$. The headpiece prevented subjects from instantaneously obtaining visual cues of the total pathway length, and it produced the sequential processing of information that constitutes an important distinction between distance cognition and distance perception (Ittelson, 1973). An initial fitting and testing procedure insured that the visual field of each subject was equally limited.

Experimental design. The design of this experiment was a 2 (frequency of names) by 3 (type of memory task) betweengroups factorial design. Three dependent variables were re-
Table 1

Proper Names Used as Intersection Labels in Experiment 1 and Frequency of Occurrence of Each per 100,000 Words in the English Language

\begin{tabular}{lcccc}
\hline \multicolumn{2}{c}{ High Frequency } & & \multicolumn{2}{c}{ Low Frequency } \\
\cline { 5 - 5 } Name & Frequency & & Name & Frequency \\
\hline Smith & 206 & & Stowe & 4 \\
Nancy & 126 & & Netta & 9 \\
Lewis & 128 & & Lowry & 2 \\
Johnson & 179 & & Jillson & 2 \\
Edward & 252 & & Elliot & 6 \\
Arthur & 117 & Alcott & 7 \\
Helen & 155 & Hilda & 7 \\
Martin & 193 & & Milrow & 4 \\
Franklin & 130 & & Fletcher & 7 \\
Charles & 237 & Crookes & 2 \\
Benjamin & 129 & Boniface & 10 \\
Wilson & 195 & Weston & 3 \\
Thomas & 244 & Talbot & 6 \\
Richard & 254 & Randall & 8 \\
Daniel & 158 & Dexter & 7 \\
\hline
\end{tabular}

Note-The source of these words was Carroll, Davies, and Richman (1971).

corded: distance estimates, memory performance, and traversal times.

Procedure. Each subject was fitted with the vision-restricting headpiece outside the laboratory room, then he or she was guided by the experimenter into the room and was positioned at the beginning of the pathway. Subjects were randomly assigned to walk either the high-or low-frequency names pathway, the intersection name placards being positioned along the pathway prior to each traversal corresponding to the treatment condition about to be administered. Instructions read to each subject at the beginning of the pathway were as follows: "You must walk along the pathway marked with masking tape on the floor. Stay on the tape at all times; if it turns, you should turn also, and continue following it until it ends. A number of other paths will intersect the path you are following, each of which is clearly labeled with a proper name. Do not turn onto any of these labeled intersecting paths. Walk at a comfortable speed along the pathway, carefully noticing the name of each intersecting path that you pass. When you reach the end of the path, you will be tested to see how many of the intersecting path names you can remember." Traversal times for each subject were unobtrusively recorded by the experimenter with a stopwatch.

Dependent variables. In the recognition and recall conditions, the experimenter first obtained an estimate of traversed distance after each subject finished walking the pathway, using a ratio estimation technique. This was accomplished by guiding the subject along a $2.2-\mathrm{m}$-long reference path located near the end of the experimental pathway. The subject was then given a $28-\mathrm{cm}$-long sheet of paper with a $3-\mathrm{cm}$ line drawn on it and was told that the line represented the length of the reference path. Next, the subject was asked to draw another line representing the length of the experimental path in the proportion to the length of the reference path. Finally, subjects in these conditions were either asked to list all of the intersection names that they could recall, in any order, or were tested for recognition of the intersection names. The recognition test was accomplished by first selecting 15 high- and 15 low-frequency distractors matched on initial letter and frequency of occurrence with the actual words along the path. Target and distractor words were typed, one each, on $3 \times 5$ in. blank index cards; the appropriate frequency-of-occurrence target and distractor cards were shuffled for each subject, then they were presented one at a time for $5 \mathrm{sec}$ to each subject. The subject responded verbally, "yes" or 
"no," to the question, "Was this word an intersection label on the path you just walked?" The experimenter recorded the response and proceeded to the next test card, until all 30 had been presented.

In the "prompt" condition, the distance estimate was not obtained immediately following the pathway traversal. Instead, subjects were shown a list with all 15 of either the high- or lowfrequency intersection names for $10 \mathrm{sec}$. The order of the words in the list matched the presentation order during the pathway traversal. Then, the distance estimate was obtained, using the same ratio estimation procedure described above.

\section{Results}

The mean distance estimates, memory performance, and traversal times for each experimental condition are displayed in Table 2. Distance estimates were obtained by measuring the lines drawn by all subjects to the nearest millimeter. Memory performance was assessed by computing the percent correct for both recall and recognition tests. Traversal time was measured to the nearest second for each pathway traversal.

Significant differences in estimated distance were observed as a function of name frequency: The pathway with high-frequency intersection names was consistently estimated as longer than the pathway with low-frequency names $[F(1,90)=30.2, p<.001]$. Report modality also exerted an influence on distance estimates $[F(2,90)=8.6, p<.01]$. Contrasts performed on this factor indicate that the significant effect was due to the influence of the prompt manipulation. A comparison of the mean distance estimated made in the prompt condition with the mean distance estimate in recall and recognition conditions indicated that prompting increased distance estimates $[F(1,90)=110.6, p<.001]$.

Analysis of distance estimates also revealed a significant interaction between name frequency and report mode $[F(2,90)=3.5, p<.05]$. This interaction was further analyzed through the comparison of simple main effects for recall, recognition, and prompt manipulations at each level of name frequency. The analysis of simple main effects revealed that high-frequency names led to longer distance estimates in the recall condition $[F(1,90)=20.1, p<.01]$ and in the recognition condition $[F(1,90)=15.75, p<.01]$. Differences between high- and low-frequency names were not, however significant in the prompt condition $[F(1,90)=1.02$, n.s.]. It appears, therefore, that the prompt manipulation attenuated the effect of intersection name memorability on distance estimation.

Analysis of the data on memory performance for each experimental condition indicated the expected effects for both word frequency and report modality. High-frequency words were remembered better than were low-frequency words $[F(1,60)=8.9, p<.01]$. Recognition performance exceeded recall performance $[F(1,60)=153.1, p<.001]$. The analysis also indicated a significant interaction between name frequency and report mode $[F(1,60)=4.1, p<.05]$. Analysis of simple main effects indicated that this interaction was due to the combination of differences in recall between low- and high-frequency words $[F(1,60)=12.2$, $p<.001]$ and the lack of difference in recognition performance between these conditions $[F(1,60)<1]$.

Analysis of the data on traversal times indicated that the experimental manipulations did not influence this variable.

\section{Discussion}

The data from Experiment 1 support the general notion that memory for route attributes is positively related to the estimated length of a traversed route. The pathway with easily memorable (high-frequency names) intersections was estimated consistently as longer than the identical pathway labeled with less memorable (low-frequency names) intersections. The data further suggest that it is retrieval of route attributes rather than the storage of route attributes that influenced distance cognition. Recognition tests, which were assumed to assess stored information, revealed no differences between low- and high-frequency name conditions. The average subject in either level of name frequency was able to recognize approximately $75 \%$ of the intersection names. Recall tests, which were assumed to reflect the additional effect of retrieval operations, indicated a substantial difference between levels of this factor. High-frequency names were significantly easier to recall than were low-frequency names. Since the difference in distance estimation parallels the difference in recall

Table 2

Results of Experiment 1, Showing Mean Distance Estimates (in Millimeters), Mean Memory Performance (Percent Correct), and Mean Traversal Time (in Seconds) as a Function of Word Frequency and Report Mode

\begin{tabular}{|c|c|c|c|c|c|c|c|c|c|}
\hline & \multicolumn{3}{|c|}{ Estimated Distance } & \multicolumn{3}{|c|}{ Memory Performance } & \multicolumn{3}{|c|}{ Traversal Time } \\
\hline & \multicolumn{2}{|c|}{ Frequency } & \multirow[b]{2}{*}{ Total } & \multicolumn{2}{|c|}{ Frequency } & \multirow[b]{2}{*}{ Total } & \multicolumn{2}{|c|}{ Frequency } & \multirow[b]{2}{*}{ Total } \\
\hline & Low & High & & Low & High & & Low & High & \\
\hline Recall & 122 & 184 & 306 & 26.7 & 42.9 & 34.8 & 45.3 & 43.1 & 88.4 \\
\hline Recognition & 148 & 203 & 351 & 73.7 & 77.1 & $75.4 * *$ & 42.1 & 41.6 & 83.7 \\
\hline Prompt & 187 & 201 & $388^{*}$ & & & & 41.1 & 39.7 & 80.8 \\
\hline Total & 457 & $588^{* *}$ & & 50.2 & $60.0^{*}$ & & 128.5 & 124.4 & \\
\hline
\end{tabular}

Note-Asterisks indicating significance levels for main effects are placed next to largest of treatment sums included in comparison. ${ }^{*} p<.01 . \quad *{ }^{*} p<.001$. 
rather than recognition, it may tentatively be assumed that retrieval of route information influenced distance estimates.

Further support for this line of reasoning is provided by the data from the "prompt" condition. It was assumed that prompting subjects would minimize the differences in recall between high- and low-frequency name conditions and, hence, minimize differences in distance estimation. This result was obtained; however, the absence of recall and recognition tests in the prompt condition renders the effect of the manipulation unclear. Prompting may have affected recall, or recognition, or both.

Experiment 2 was designed to explore the retrieval hypothesis more fully by systematically employing prompts and assessing their influence on both memory performance and distance estimation. In a procedure similar to that used in Experiment 1, subjects traversed a pathway with 15 labeled intersections. In this experiment, intersection names were the same for all subjects. Intersections were labeled with 15 names that consisted of 3 names from each of five superordinate categories. Half of the subjects were assigned to a prompt condition, in which the five superordinate category names were presented prior to distance estimation. The remainder of the subjects were not prompted. Memory tests followed the distance estimation task in order to assess the effects of the prompts. It was assumed that category prompts would facilitate recall but not recognition (Cohen, 1966; Tulving \& Pearlstone, 1966). If the expected difference in recall was paralleled by a difference in distance estimation, the pattern of data would support the hypothesis that the retrieval of route information is positively related to the estimation of traversed distance.

\section{EXPERIMENT 2}

\section{Method}

Subjects. Subjects were 48 introductory and environmental psychology students at Arizona State University who partially fulfilled a course requirement by participating in this experiment. Approximately equal numbers of males and females served as subjects in a between-subjects design.

Apparatus. The pathway used in this study was the same as in Experiment 1; the same vision-restricting headpiece was also used. Intersection labels for this experiment were selected from the set of "basic objects" names described by Rosch, Mervis, Gray, Johnson, and Boyes-Braem (1976). Three instances of each of five superordinate categories were represented along the pathway. Table 3 shows the words used as intersection labels in Experiment 2 and the categories to which they belong.

The labels were lettered on pieces of posterboard as in the first experiment and placed adjacent to the pathway. The order was determined using a random number program, and it was maintained throughout the experiment.

Experimental design. The design of this experiment was a 1 (presence of prompt) by 2 (type of memory task) betweengroups factorial design. Three dependent variables, distance estimation, memory performance, and traversal time, were employed.
Table 3

Basic Object Names Used as Intersection Labels in Experiment 2 and the Corresponding Superordinate Categories Used as Prompts

\begin{tabular}{ll}
$\begin{array}{c}\text { Basic Object } \\
\text { Names }\end{array}$ & $\begin{array}{c}\text { Superordinate } \\
\text { Categories }\end{array}$ \\
\hline $\begin{array}{l}\text { Chair } \\
\text { Lamp } \\
\text { Table } \\
\text { Salmon }\end{array}$ & Furniture \\
Trout & \\
Bass & Fish \\
Shirt & \\
Socks & \\
Pants & Clothing \\
Piano & \\
Guitar & \\
Drum & Musical Instruments \\
Peach & \\
Apple & \\
Grape & Fruit \\
\hline
\end{tabular}

Procedure. The procedure followed in this experiment, including instructions delivered to the subjects, was identical to that described in Experiment 1 up to the point that the pathway traversal was completed, and the traversal time was unobtrusively recorded.

Subjects were randomly assigned to either a prompt or a no-prompt condition before they arrived at the experiment. Subjects in the former condition were presented with a category prompt immediately following the pathway traversal, prior to distance estimation and memory testing. Next, distance estimates for the traversed pathway were completed using the same ratio estimation technique described in Experiment 1. Finally, the memory measure was obtained, with each subject performing either a recall or a recognition test in the same manner as described in Experiment 1.

\section{Results}

The mean distance estimates, memory performance, and traversal times for each experimental condition are displayed in Table 4. Distance estimates were recorded by measuring the lines drawn by each subject to the nearest millimeter. Memory performance was assessed by computing the percent correct for both recall and recognition tests. Traversal time was recorded to the nearest second for each pathway traversal.

Separate 2 by 2 analyses of variance were employed to analyze each dependent variable. These analyses indicated a significant main effect of the prompt manipulation on distance estimation: Prompting reliably increased the perceived length of the pathway $[F(1,44)=6.1, p<.025]$. Prompting also increased the number of intersection names remembered $[F(1,44)=$ $6.9, \mathrm{p}<.025]$. The expected difference in memory performance between recognition and recall measures was noted; subjects were able to recognize substantially more intersection names than they were able to recall $[F(1,44)=27.7, p<.001]$. Report modality had no effect on distance estimates, nor was there an interaction between prompting and report modality on the 
Table 4

Results of Experiment 2, Showing Mean Distance Estimates (in Millimeters) and Mean Memory Performance (Percent Correct) as a Function of Report Mode and the Presence of a Category Prompt, and Mean Traversal Times for the Various Conditions

\begin{tabular}{|c|c|c|c|c|c|c|c|c|c|}
\hline & \multicolumn{3}{|c|}{ Estimated Distance } & \multicolumn{3}{|c|}{ Memory Performance } & \multicolumn{3}{|c|}{ Traversal Time } \\
\hline & Prompt & No Prompt & Total & Prompt & No Prompt & Total & Prompt & No Prompt & Total \\
\hline $\begin{array}{l}\text { Recall } \\
\text { Reconnition }\end{array}$ & $\begin{array}{l}223 \\
196\end{array}$ & $\begin{array}{l}188 \\
184\end{array}$ & $\begin{array}{l}411 \\
380\end{array}$ & $\begin{array}{l}58.9 \\
70.0\end{array}$ & $\begin{array}{l}40.0 \\
68.9\end{array}$ & $\begin{array}{l}49.5 \\
69.5 * *\end{array}$ & $\begin{array}{l}45.7 \\
46.4\end{array}$ & $\begin{array}{l}45.9 \\
50.0\end{array}$ & $\begin{array}{l}91.6 \\
96.4\end{array}$ \\
\hline Total & $419 *$ & 372 & & $64.5 *$ & 54.5 & & 92.1 & 95.9 & \\
\hline
\end{tabular}

Note-Asterisks indicating significance levels for main effects are placed next to largest of treatment sums included in comparison. ${ }^{*} p<.025 . \quad{ }^{* *} p<.001$.

distance estimation variable. Analysis of the traversal time data indicated that none of the experimental manipulations affected traversal time.

The analysis of variance performed on memory tasks indicated a significant interaction between the prompt manipulation and the report modality manipulation $[F(1,44)=5.5, p<.025]$. An analysis of simple main effects indicated that the prompt manipulation strongly affected recall performance $[F(1,44)=12.4, p<.005]$ but failed to influence recognition performance $[F(1,44)<1]$. Recall performance was significantly improved, approaching the level of recognition performance, by the prompt manipulation.

\section{Discussion}

The results of Experiment 2 provide further evidence for the hypothesis that the retrieval of route information nfluences the estimation of route length. Providing superordinate category prompts for intersection names ncreased the number of names that could be recalled. This result replicates the finding of Jablonski (1972) and Lewis (Note 1) that cued recall is superior to uncued ecall. Cuing with superordinate category names is assumed to increase recall by facilitating retrieval of nformation from storage (Kausler, 1974, p. 369). In he present study, providing retrieval cues simultanesusly increased information recall and estimates of route ength. Prompting did not, however, increase the number of intersection names that could be recognized.

The data suggest the following interpretation. While raversing a pathway, subjects attend to, encode, and itore information about the route. When subjects are iubsequently asked to make a distance estimate, a nemory search is instigated during which pathway. elated information is sought. Within limits, the more nformation that can be retrieved regarding pathway tructure, the longer the pathway is estimated. Promptng subjects following pathway traversal increased the ccessibility of stored route information and hence roduced the observed effect on distance estimates.

In both experiments reported here, observed diferences in distance estimates between experimental onditions may not be attributed to actual time required o traverse the pathway, although the experimental nanipulations may have affected estimated time of traversal. Parenthetically, the nearly identical mean traversal times between conditions also provides some assurance that the presentation rate of the to-beremembered intersection names was the same for all conditions.

The present research does not test the hypothesis that the amount of stored information affects the cognition of route length. In the two studies reported above, information in storage was held constant, while information accessibility was varied. Manipulations that increased the amount of retrievable information were observed to increase estimates of route length. It should be noted, however, that this research does not rule out the validity of the "storage" hypothesis. The storage hypothesis might be further examined using a design in which the amount of information in storage is varied, while the amount of retrievable information is held constant. It remains possible that distance estimates are a function of both stored and accessed information.

The retrieval hypothesis provides an information processing explanation for previous findings relating distance estimates to the structural properties of a route. As noted above, estimated route length has been found to increase with increases in the number of tums or intersections that are distributed along a route. Perceived route length is also influenced by the complexity of a route: Structurally complex routes are estimated as longer than simple routes (Walmsley, 1978). A number of researchers have also suggested that subjective distance is exaggerated relative to objective distance in areas such as city centers where "there is a denser packing of land uses" (Golledge, Briggs, \& Demko, 1969 , p. 61). Explanations of this effect have focused on two factors: the increased number of intervening nodes between two locations, and the experience of increased travel times. The present research indicates that information pertaining to traversed routes increases the subjective length of those routes to the extent that it is easily retrievable.

\section{REFERENCE NOTE}

1. Lewis, M. Q. Cue effectiveness in cued recall. Paper presented at the annual meeting of the Psychonomic Society, Saint Louis, November 1972. 


\section{REFERENCES}

Carroll, J., Davies, P., \& Richman, B. The American Heritage word frequency book. Boston: Houghton-Mifflin, 1971.

Conen, B. Some-or-none characteristics of coding behavior. Journal of Verbal Learning and Verbal Behavior, 1966, 5, 182-187.

DEEse, J. Frequency of usage and number of words in free recall: The role of association. Psychological Reports, 1960 , 7, 337-344.

Downs, R., \& SteA, D. Image and environment. Chicago: Aldine, 1973.

Downs, R., \& STEA, D. Maps in minds: Reflections on cognitive mapping. New York: Harper \& Row, 1977.

Golledge, R. G., Briggs, R., \& Demko, D. The configuration of distances in intra-urban space. Proceedings, Association of American Geographers, 1969, 1, 60-65.

Gould, P., \& White, R. Mental maps. Baltimore: Penguin Books, 1974.

HaLL, J. Learning as a function of word frequency. American Journal of Psychology, 1954, 67, 138-140.

ITTELSON, W. H. Environment perception and contemporary perceptual theory. In W. H. Ittelson (Ed.), Environment and cognition. New York: Seminary Press, 1973.

JABLONSKI, E. M. Cued and uncued multitrial free recall as a function of age, mnemonic instruction, and sex. Unpublished doctoral dissertation, University of Missouri, 1972.

KaUSLER, D. H. Continuity of processes across variants of recognition learning. In R. L. Solso (Ed.), Theories of cognitive psychology: The Loyola symposium. Potomac, Md: Erlbaum, 1974.

KInTsCh, W. Learning, memory, and conceptual processes. New York: Wiley, 1970.

Kosslyn, S. M., Pick, H. L., \& Fariello, G. R. Cognitive maps in children and man. Child Development, 1974, 45, 707-716.
LEE, T. Perceived distance as a function of direction in a city. Environment and Behavior, 1970, 2, 40-51.

Milgram, S. Chapter Il, Introduction. In W. H. Ittelson (Ed.), Environment and cognition. New York: Seminary Press, 1973.

Ornstein, R. On the experience of time. Baltimore: Penguin Books, 1969.

Rosch, E., Mervis, C., Gray, W., Johnson, D., \& BoyesBraem, P. Basic objects in natural categories. Cognitive Psychology, 1976, 8, 382-439.

Sadalla, E., \& Magel, S. The perception of traversed distance. Environment and Behavior, in press.

Sadalla, E., \& Staplin, L. The perception of traversed distance: Intersections. Environment and Behavior, in press.

Siegel, A. W., \& White, S. H. The development of spatial representations of large-scale environments. In C. Reese (Ed.), Advances in Child Development and Behavior, 1975, 10, 10-55.

Slamecka, N. J. An examination of trace storage in free recall. Journal of Experimental Psychology, 1968, 76, 504-513.

STEA, D. The measurement of mental maps: An experimental model for studying conceptual spaces. In K. Cox \& R. Golledge (Eds.), Behavioral problems in geography: A symposium. Evanston, Ill: Northwestern University Press, 1969.

Tulving, E., \& Osler, S. Effectiveness of retrieval cues in memory for words. Journal of Experimental Psychology, 1968, 77, 593-601.

Tulving, E., \& Pearlstone, Z. Availability versus accessibility of information in memory for words. Journal of Verbal Learning and Verbal Behavior, 1966, 5, 381-391.

Walmsley, D. Stimulus complexity in distance distortion. Professional Geographer, 1978, 30, 14-19.

(Received for publication August 3, 1978; revision accepted May $8,1979$. 